\title{
Reconfiguration of a Vertebrate Motor Network: Specific Neuron Recruitment and Context-Dependent Synaptic Plasticity
}

\author{
Wen-Chang Li, ${ }^{1}$ Bart Sautois, ${ }^{2}$ Alan Roberts, ${ }^{1}$ and Stephen R. Soffe ${ }^{1}$ \\ ${ }^{1}$ School of Biological Sciences, University of Bristol, Bristol BS8 1UG, United Kingdom, and ${ }^{2}$ Department of Applied Mathematics and Computer Science, \\ Ghent University, B-9000 Ghent, Belgium
}

\begin{abstract}
Motor networks typically generate several related output patterns or gaits where individual neurons may be shared or recruited between patterns. We investigate how a vertebrate locomotor network is reconfigured to produce a second rhythmic motor pattern, defining the detailed pattern of neuronal recruitment and consequent changes in the mechanism for rhythm generation. Hatchling Xenopus tadpoles swim if touched, but when held make slower, stronger, struggling movements. In immobilized tadpoles, a brief current pulse to the skin initiates swimming, whereas $40 \mathrm{~Hz}$ pulses produce struggling. The classes of neurons active during struggling are defined using whole-cell patch recordings from hindbrain and spinal cord neurons during $40 \mathrm{~Hz}$ stimulation of the skin. Some motoneurons and inhibitory interneurons are active in both swimming and struggling, but more neurons are recruited within these classes during struggling. In addition, and in contrast to a previous study, we describe two new classes of excitatory interneuron specifically recruited during struggling and define their properties and synaptic connections. We then explore mechanisms that generate struggling by building a network model incorporating these new neurons. As well as the recruitment of new neuron classes, we show that reconfiguration of the locomotor network to the struggling central pattern generator (CPG) reveals a context-dependent synaptic depression of reciprocal inhibition: the result of increased inhibitory neuron firing frequency during struggling. This provides one possible mechanism for burst termination not seen in the swimming CPG. The direct demonstration of depression in reciprocal inhibition confirms a key element of Brown's (1911) hypothesis for locomotor rhythmogenesis.
\end{abstract}

Key words: Xenopus; synaptic depression; interneurons; modeling; switching; inhibition

\section{Introduction}

Most animals show multiple motor patterns for respiration (panting, sighing), feeding (biting, swallowing), and locomotion (walking, trotting). Evidence from invertebrates shows that different patterns can share circuit elements through a process of network reconfiguration (Getting and Dekin, 1985; Getting, 1989; Meyrand et al., 1991; Jing and Weiss, 2002; Popescu and Frost, 2002; Marder et al., 2005; Briggman and Kristan, 2006). The observation that individual neurons can participate in more than one pattern has suggested that the same process occurs in vertebrates, but experimental evidence remains patchy (Berkinblit et al., 1978; Carter and Smith, 1986; Bekoff et al., 1987; Currie and Stein, 1989; Mortin and Stein, 1989; Soffe, 1993; Lieske et al., 2000; Berkowitz, 2002, 2005). In addition to shared elements, selective recruitment of neurons between patterns also occurs in larval zebrafish and turtles (Ritter et al., 2001; Kimura et al., 2006;

Received July 9, 2007; revised Sept. 11, 2007; accepted Sept. 11, 2007.

This work was supported by the Wellcome Trust. We thank Erin Anderson, Tim Colborn, Emma Hanmore, and Jenny Maxwell for technical help. Bart Sautois is a research assistant of the Research Foundation-Flanders (FW0Vlaanderen). Wen-Chang Li is a Royal Society University Research Fellow.

Correspondence should be addressed to Dr. Stephen R. Soffe, School of Biological Sciences, University of Bristol, Woodland Road, Bristol BS8 1UG, UK. E-mail: s.r.soffe@bristol.ac.uk.

D01:10.1523/JNEUROSCI.3694-07.2007

Copyright $\odot 2007$ Society for Neuroscience $\quad 0270-6474 / 07 / 2712267-10 \$ 15.00 / 0$
Berkowitz, 2007). However, in most vertebrates, too little is known about the neurons involved to determine whether recruitment for a particular pattern occurs within neuronal classes, perhaps with modulation of cellular properties, or whether recruitment introduces fundamentally different neuron types with intrinsically different properties. Additionally, little is known about how the mechanisms generating different patterns change after reconfiguration.

Because vertebrates share common ancestry, the basis of motor pattern selection has been successfully explored in lower vertebrates like zebrafish (Ritter et al., 2001), turtles (Stein, 2005) and young frog tadpoles (Soffe, 1991, 1993, 1996, 1997). Hatchling Xenopus tadpoles select between two main responses: swimming if touched, with low-amplitude $12-25 \mathrm{~Hz}$ alternating bends passing from head to tail (Kahn et al., 1982); struggling if held, with stronger $2-10 \mathrm{~Hz}$ bends passing from tail to head (Kahn and Roberts, 1982). In immobilized tadpoles, single electrical pulses to the trunk skin excite the nerve endings of touch-sensitive sensory Rohon-Beard (RB) neurons to start swimming (Clarke et al., 1984), whereas similar pulses at $40 \mathrm{~Hz}$ produce struggling (Soffe, 1991). Our aim was to uncover the cellular mechanisms that allow these different patterns of sensory activity to select and generate appropriate motor patterns for swimming and struggling. 
It was originally concluded that a switch from swimming to struggling involved recruitment of neurons only from within the neuron classes that generate swimming (Soffe, 1993). To reevaluate this previous conclusion, we used whole-cell recording to define classes of hindbrain and spinal cord neurons that are active during struggling evoked by $40 \mathrm{~Hz}$ stimulation of the skin. We found two new classes of excitatory neuron specifically recruited during struggling and investigated their properties and synaptic connections. The switch from swimming to struggling also involves a change from a pattern of alternating single spikes to one of alternating bursts introducing a critical requirement for a burst termination mechanism. We have investigated this mechanism using physiology and computer modeling, building a network model incorporating the new neuron classes recruited during struggling. Specifically, we explored the proposal made by Brown (1911) that synaptic depression of reciprocal inhibition plays an important role in burst termination after reconfiguration to a struggling central pattern generator (CPG).

\section{Materials and Methods}

Details of the whole-cell patch recording methods have been given previously (Li et al., 2002). Briefly, Xenopus tadpoles at stage 37/38 were anesthetized with $0.1 \% 3$-aminobenzoic acid ester (MS-222; Sigma, Poole, UK), immobilized in $10 \mu \mathrm{M} \alpha$-bungarotoxin saline, then pinned in a bath of saline [containing (in $\mathrm{mm}$ ): $115 \mathrm{NaCl}, 3 \mathrm{KCl}, 2 \mathrm{CaCl}_{2}, 1$ $\mathrm{MgCl}_{2}, 2.4 \mathrm{NaHCO}_{3}$, and $10 \mathrm{HEPES}$, adjusted with $5 \mathrm{M} \mathrm{NaOH}$ to $\left.\mathrm{pH} 7.4\right]$. In some experiments, the $\mathrm{Mg}^{2+}$ was replaced with an additional $1 \mathrm{~mm}$ $\mathrm{CaCl}_{2}$. This $0 \mathrm{~mm} \mathrm{Mg}^{2+}$ saline was used so NMDAR-mediated components could be seen. Skin and muscles over the left side of the spinal cord were removed and a mid-dorsal cut made along the spinal cord to open the neurocoel. Small cuts were made in the wall of the neurocoel on the right side to expose more ventral neurons. The tadpole was then repinned in a small $2 \mathrm{ml}$ recording chamber with saline flow of $\sim 2 \mathrm{ml}$ per minute. Exposed neuronal cell bodies were seen using a $40 \times$ waterimmersion lens with bright field illumination on an upright Nikon (Tokyo, Japan) E600FN microscope. Antagonists were dropped into a $200 \mu \mathrm{l}$ well upstream to the recording chamber. Drugs used were 2,3dihydroxy-6-nitro-7-sulfamoylbenzo- $[f]$ quinoxaline- $[f]$ quinoxaline (NBQX; Tocris, Ellisville, MO), D-(-)-2-amino-5-phosphonopentanoicacid (D-AP5; Tocris), and dihydro- $\beta$-erythroidine (DH $\beta$ E; Research Biochemicals International, Natick, MA).

Patch pipettes were filled with $0.1 \%$ neurobiotin and $0.1 \%$ Alexa Fluor 488 (Invitrogen, Eugene, OR) in intracellular solution [containing (in $\mathrm{mm}$ ): $100 \mathrm{~K}$-gluconate, $2 \mathrm{MgCl}_{2}, 10$ EGTA, 10 HEPES, $3 \mathrm{Na}_{2} \mathrm{ATP}$, and 0.5 $\mathrm{NaGTP}$, adjusted to $\mathrm{pH} 7.3$ with $\mathrm{KOH}$ ) and had resistances between 10 and $20 \mathrm{M} \Omega$. Junction potentials were corrected before making recordings. Signals were recorded with an Axoclamp 2B in conventional bridge or continuous single-electrode voltage-clamp mode, acquired with Signal software through a CED 1401 Plus interface with a sampling rate of 10 $\mathrm{kHz}$. Off-line analyses were made with Minitab and Excel. All values are given as mean \pm SD. Statistical analysis was by two-sample $t$ test unless stated otherwise. Experiments comply with the United Kingdom Home Office regulations and have received local ethical approval.

Neuron anatomy was revealed by filling with neurobiotin diffused through recording patch pipettes. After fixing and processing, the CNS was exposed and specimens mounted on their sides between coverslips for observation, tracing with a drawing tube, or photography at $200 \times$ on a bright field microscope (Li et al., 2002). All measures on fixed specimens were multiplied by 1.28 to compensate for shrinkage during dehydration (Li et al., 2001).

For network modeling, the neurons that are active during swimming [ascending interneurons (aINs), commissural interneurons (cINs), and motoneurons (mns)] were modeled using the individual single compartment neuron models described by Sautois et al. (2007). The new type of neuron used in the network model [repetitive-firing descending interneuron $(\mathrm{dIN} r)$ ] was modeled using the same Hodgkin-Huxley type equations, but with new parameter values based on our measurements (supplemental Table 2, available at www.jneurosci.org as supplemental material). The equations and parameters for synapses were taken from (Sautois et al., 2007); the model uses specific opening and closing rates for conductances at each type of synapse. To avoid artificial synchrony in the network output, some variability (in the order of 5\%) was added to the excitatory connections from driver neurons (see below) to dIN $r$ s, and to those from dIN $r$ s to mns and cINs.

The network model was based on a pair of half centers, each containing three of each type of CPG neuron ( $\mathrm{dIN} r$, cIN, aIN, and $\mathrm{mn}$ ). To imitate the effects of continuous stimulation of the skin on both sides of the body, the simplified sensory activation pathway consisted of one neuron on each side, exciting CPG neurons through synapses activating NMDARs with an infinite closing time constant. Thus, each side received constant excitation. Synaptic depression was modeled using a series of terms that recovered exponentially after each previous spike. Each term involved two parameters: a synaptic plasticity factor $(\alpha)$, setting the extent of depression, and the time constant of recovery $(\beta)$. The sum of these terms was used to alter the synaptic conductance. (For details, see below and supplemental material, available at www.jneurosci.org.)

\section{Results}

The introduction of whole-cell patch recordings from pairs of neurons labeled anatomically with neurobiotin has meant that our ability to characterize neurons in the hatchling Xenopus tadpole has advanced considerably since previous investigations of the struggling network (Soffe, 1993, 1996). As well as making possible a more confident definition of neuron classes based on both physiology and anatomy, it has also provided new data on the specific firing properties of each neuron class (Li et al., 2002, 2004a, 2006; Sautois et al., 2007). Our previous knowledge of cellular properties was primarily based on sharp electrode recordings from unidentified ventral neurons presumed to be motoneurons (Soffe, 1990). This study uses our ability to define neuron classes to focus on the way the swimming network is reconfigured to produce a slower pattern of rhythmic motor bursts suitable for struggling. It does not investigate the reversed, tail-to-head spread of activity during struggling (Kahn and Roberts, 1982; Soffe, 1991), and we do not consider longitudinal aspects of reconfiguration.

\section{A commissural sensory pathway for the activation of struggling}

Electrical stimulation to the trunk skin on one side of the body at $40 \mathrm{~Hz}$ imitates applied pressure and can lead to struggling activity in the motoneurons on both sides (Soffe, 1991). This motoneuron activity during struggling can be seen in ventral root recordings (Fig. $1 B, D$ ), which show high-frequency bursts occurring at periods ranging from 100 to $500 \mathrm{~ms}$ (Kahn and Roberts, 1982; Soffe, 1991, 1993). During this type of stimulation, the known commissural sensory pathway dorsolateral commissural (dlc) INs fire at short latency, but are then inhibited (Soffe, 1993). They receive glycinergic inhibition from vigorously active aINs ( $\mathrm{Li}$ et al., 2002, 2004a). If these dlc INs, which provide excitation to the other side to trigger swimming, are silent, are other commissural neurons recruited to carry excitation to the other side to drive struggling symmetrically?

To find candidate commissural neurons, we used loose-patch electrodes, in the dorsolateral region of the rostral spinal cord where sensory interneurons lie, to search for neurons excited by $40 \mathrm{~Hz}$ stimulation of the skin on the same side. Once these candidates were found, a whole-cell patch recording was made from a neuron on the opposite side that was active during struggling evoked by $40 \mathrm{~Hz}$ stimulation. The candidate commissural neurons were then stimulated one-by-one with the loose-patch elec- 
A
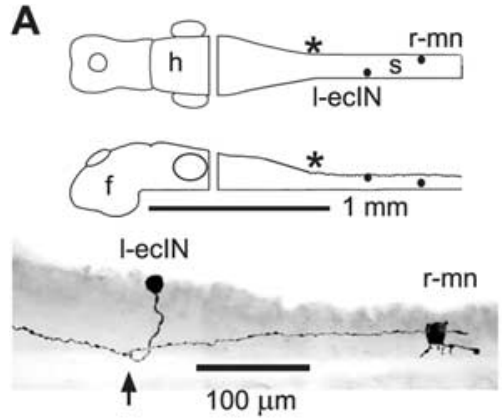

C
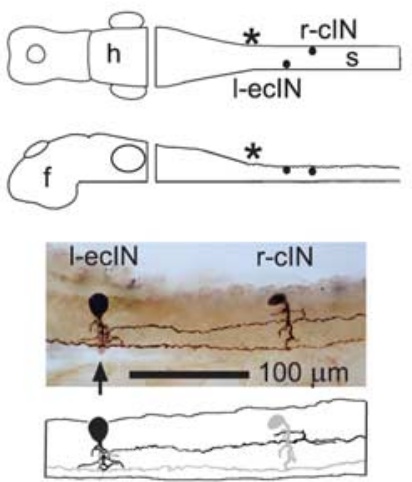

E $15 p A$

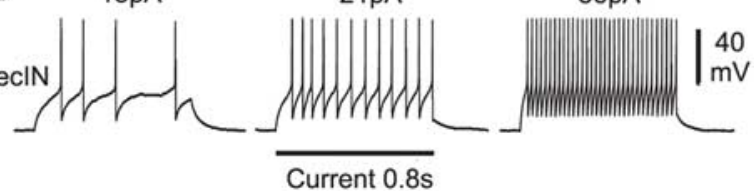

B

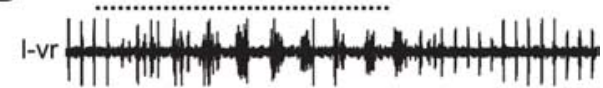
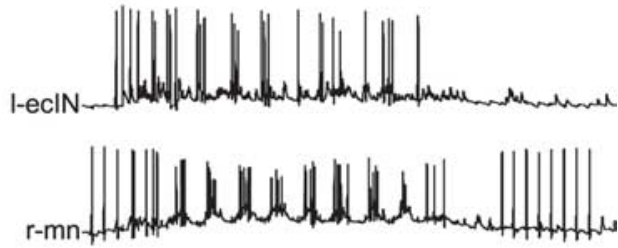

D

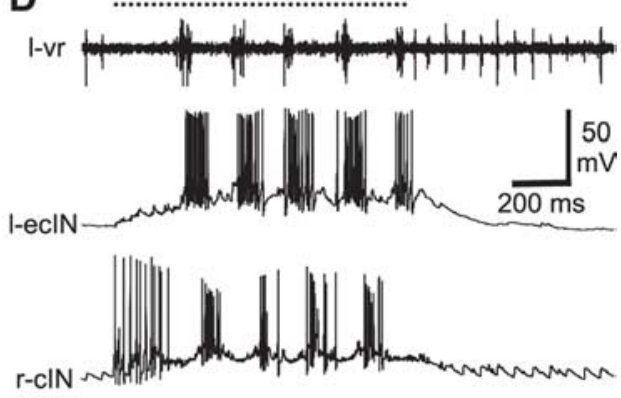

Figure 1. Anatomy and activity of ecINs and the contralateral neurons they excite. $\boldsymbol{A}$, Diagram of the CNS in dorsal and lateral view to show the location of neurons. $f$, Forebrain; $h$, hindbrain; $s$, spinal cord. An asterisk indicates the approximate position of the obex. Photomontage of neurobiotin-filled neurons in lateral view where the left-side ecIN has a ventral axon that crosses (at arrow) to contact a motoneuron ( $\mathrm{r}-\mathrm{mn}$ ) on the right side. $\boldsymbol{B}$, Activity during struggling evoked by $40 \mathrm{~Hz}$ stimulation of the trunk skin (dotted line), followed by swimming. Recording of a left-side ventral root (l-vr) with the ecIN and motoneuron from $A$. Only the motoneuron fires during swimming. Stimulus artifacts in this and subsequent figures are removed from the vr record for clarity. The ecIN made a monosynaptic connection to the $\mathrm{mn}$ (data not shown). C, Diagram, photomontage, and tracing showing a left-side ecIN with a ventral axon that crosses (at arrow) to contact a right-side cIN. D, Both neurons are vigorously rhythmically active during struggling and become silent during swimming. $\boldsymbol{E}$, Long current injections lead to repetitive firing of the ecIN. $\boldsymbol{F}$, Action potentials in the ecIN, produced by current injection, lead to EPSPS at constant latency in the cIN (seven traces overlapped; neurons from $C, D)$.

trode to see if they excited the contralateral neuron. Where connections were found, the presynaptic neuron was then recorded and stimulated in whole-cell mode and finally the anatomy of both neurons was revealed by neurobiotin injection (Fig. 1).

Paired, whole-cell recordings were made in 47 tadpoles from 53 dorsolateral interneurons, which had commissural axons and were excited to fire during $40 \mathrm{~Hz}$ skin stimulation. Each was recorded with a contralateral neuron that was rhythmically active during struggling. From these, we identified 18 neurons that produced excitation of the contralateral neuron. We call these excitatory commissural interneurons (ecINs) and they had the following features.

During swimming, ecINs received very weak, rhythmic synaptic input, but did not fire (Figs. $1 B, D, 2 B$ ). However, they were excited to fire action potentials during periods of $40 \mathrm{~Hz}$ skin stimulation that led to struggling: some fired rhythmically (Figs. $1 B, D, 2 B)$, others more tonically. These neurons are therefore selectively recruited during struggling.

They had resting potentials of $-57 \pm 8 \mathrm{mV}(n=18)$, relatively high input resistances $(2.1 \pm 0.9 \mathrm{G} \Omega ; n=14)$, and when depo-

showed clear summation (Fig. $2 F$ ).

larizing current was injected they fired repetitive action potentials with little adaptation, particularly at higher frequencies (Fig. $1 E$, supplemental Table 1, available at www.jneurosci.org as supplemental material).

Current induced ecIN action potentials led to direct, short-latency excitation of the contralateral neurons (Figs. $1 F, 2 C$ ) (latency, $1.2 \pm 0.4 \mathrm{~ms}$; range, $0.8-2.3 \mathrm{~ms}$; amplitude, $2.6 \pm 1.6 \mathrm{mV}$; range, $0.8-6.3$ $\mathrm{mV} ; n=18$ pairs sampled). In the tadpole, with fine unmyelinated axons, latencies $<3 \mathrm{~ms}$ in neurons $<300 \mu \mathrm{m}$ apart are likely to be monosynaptic ( $\mathrm{Li}$ et al., 2004b). The contralateral postsynaptic neurons were identified anatomically as mns $(n=5)$, reciprocal inhibitory cINs $(n=11)$ and ecINs $(n=2)$.

In nine cases, the synaptic connections of the ecINs were investigated in more detail. Their excitation of contralateral neurons was blocked by the joint application of the AMPAR antagonist NBQX and the NMDAR antagonist D-AP5 (Fig. 2D) $(n=$ 5) or, in $1 \mathrm{mM} \mathrm{Mg}^{2+}$ saline, by NBQX alone $(n=4)$. This suggests that they release glutamate like the other sensory pathway interneurons (Li et al., 2003).

Stimulation of the ipsilateral skin produced excitation at latencies of $3.5-15 \mathrm{~ms}$ (Fig. 2E). We should expect latencies of 7-13 ms to be disynaptic (Li et al., 2004a) (Fig. 2E, shading). However, most latencies were shorter than this, indicating that ecINs typically receive monosynaptic excitation from ipsilateral sensory RohonBeard neurons responding to skin stimulation. Unlike the strong excitation of dlc sensory interneurons (Li et al., 2003), single skin stimuli frequently failed to evoke spikes in ecINs, but during $40 \mathrm{~Hz}$ stimulation of the skin, excitation typically

All ecINs were recorded in the spinal cord between 1 and 1.2 $\mathrm{mm}$ from the midbrain (Fig. $2 G$ ) (the hindbrain ends at the obex which is $0.8-0.9 \mathrm{~mm}$ from the midbrain). Neurobiotin filling showed that they had dorsolateral somata $15-23 \mu \mathrm{m}$ in diameter with a ventrally directed axon. Dendrites were very variable. A few emerged directly from the soma, some came from the initial segment of the axon, and some emerged more ventrally. Axons crossed in the ventral commissure then branched contralaterally to ascend and descend. In 3 of 14 cases, there was no ascending axon. The mean contralateral axon length was $1.13 \pm 0.35 \mathrm{~mm}$ $(n=14)$.

In a previous study, it was concluded that only neuron types active during swimming participate in struggling (Soffe, 1993). This conclusion was based on the explicit assumption that all the spinal neuron classes were known. Our definition of ecINs as a distinct neuron class specifically recruited during struggling shows that both the conclusion and the assumption were incorrect. Although the previous study acknowledged the need for a route by which Rohon-Beard neurons could excite neurons on 
the opposite side to maintain struggling during repetitive stimulation, suitable neurons were not found. The most likely reason that the earlier study failed to distinguish the ecINs as a class is simply that with the techniques then available, many of the neurons investigated did not receive the detailed anatomical and physiological definition that has been possible here.

\section{Neurons active during struggling}

We have defined a new class of spinal sensory pathway commissural interneurons (ecINs) that are selectively recruited to fire during repetitive skin stimulation that evokes struggling and produce glutamate mediated excitation of contralateral neurons. Our next objective was to use wholecell recordings and neurobiotin filling to survey the activity of swimming CPG neurons during struggling. These were identified by a combination of documented anatomical and physiological characteristics (Dale, 1985; Roberts et al., 1999; Li et al., 2004d, 2006). Motoneurons ( $n=15$ in 15 tadpoles) (Figs. $1 B, 3 A, 4 E$ ) and reciprocal inhibitory cINs ( $n=19$ in 16 tadpoles) (Fig. $1 D, 2 B, 3 B$ ) that were active during swimming were also vigorously active in struggling. They fired bursts of up to nine spikes per cycle at instantaneous frequencies up to $245 \mathrm{~Hz}$ (mns, $104 \pm 49 \mathrm{~Hz}, n=$ 12 ; cINs, $123 \pm 34 \mathrm{~Hz}, n=15)$. Some cINs that were unreliable or silent during swimming were recruited in struggling (Fig. $1 D)$. We have shown previously that recurrent inhibitory aINs (Li et al., 2002, 2004d), which are only weakly active during swimming, also become vigorously active in struggling. This was confirmed here ( $n=6$ in six tadpoles) (Fig. $3 C$ ). These three neuron classes therefore contribute to the neuronal circuits for both swimming and struggling with some recruitment occurring within these classes during struggling.

We expected to find a similar pattern of increased firing during struggling in the excitatory spinal and reticulospinal descending interneurons (dINs and hdINs) that drive swimming. These neurons, which can be recognized in recordings by their long-duration action potentials and anatomically by their ipsilateral descending axon, are reliably active during swimming (Li et al., 2006). Surprisingly, they were only weakly active or even silent during struggling (Fig. 3D); typically, dINs fired $<1$ spike per cycle and only exceptionally up to three spikes ( $n=30$ neurons). However, we found a new physiological subclass of interneurons in the hindbrain and rostral spinal cord with ipsilateral descending axons that are selectively recruited during struggling. This subclass was not recognized in a previous study of struggling (Soffe, 1993) where the data were insufficient to show that the descending interneurons are not a homogeneous group. As described below, these new descending interneurons are distinct from the dINs that are active during swimming be-
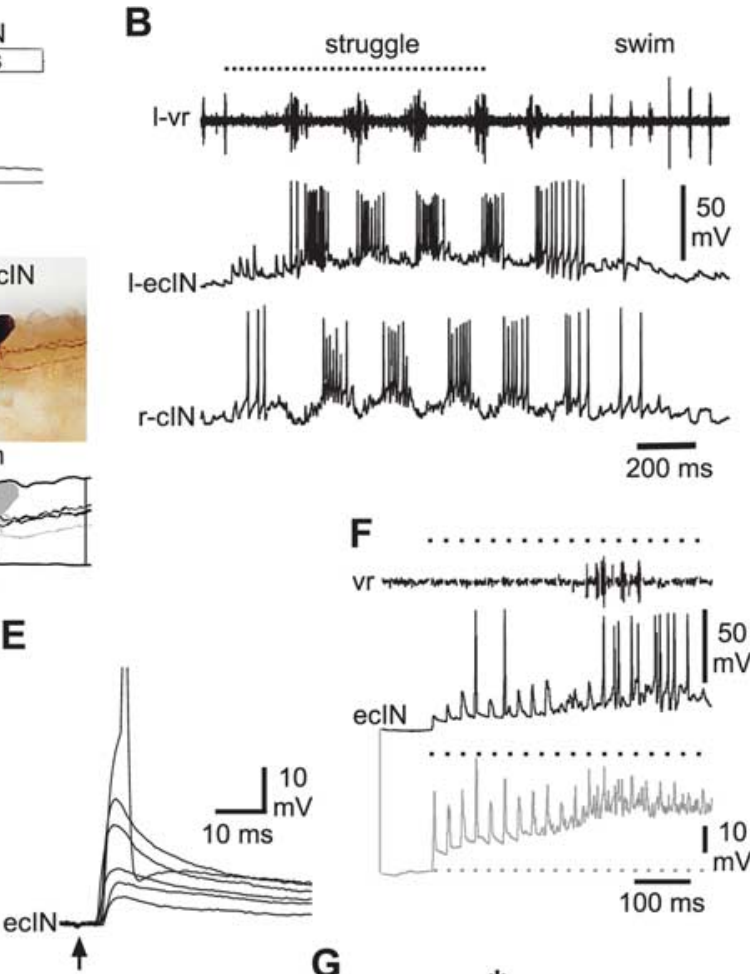
ecIN

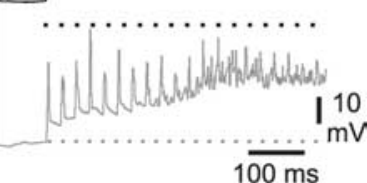

G
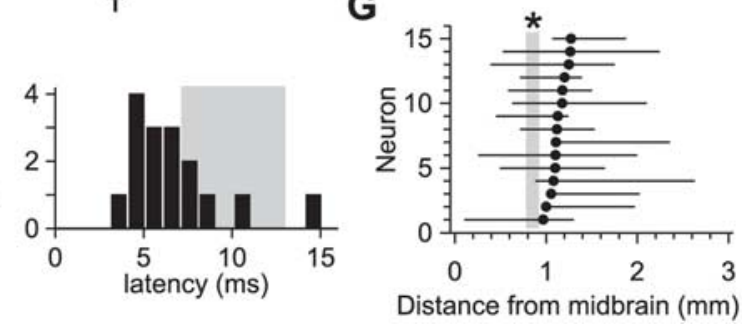

Distance from midbrain $(\mathrm{mm})$

Figure 2. Synaptic connections to and from an ecIN. $\boldsymbol{A}$, Diagrams, photomontage, and tracing showing a left-side ecIN with a ventral axon that crosses (at arrow) to contact a commissural interneuron on right side (cIN). $\boldsymbol{B}$, Recording of left-side ventral root injection. $\boldsymbol{D}$. The EPSPs are reversibly blocked by the glutamate antagonists NBQX (2.5 $\mu \mathrm{m})$ and D-AP5 (25 $\mu \mathrm{m})$ (averages of five (thaces). $\boldsymbol{E}$, Increasing stimulation of the rostral trunk skin (arrow) on the same side as the ecIN evokes EPSPs at short latency which trong summation (gray dots indicate resting potential). G, Longitudinal soma locations for recorded ecINs (black dots) and their contralateral axon projections (lines). The gray bar and asterisk indicate the approximate position of the obex.

cause they have a shorter duration action potential and fire repetitively when depolarized by current injection. On the basis of this latter property, we have termed them $\mathrm{dINr}$ (repetitive-firing dINs). Unlike dINs, the dIN rs were typically silent during swimming but became vigorously active during struggling (Fig. 3E).

\section{Neurons producing descending excitation for struggling: dIN $r$}

The dINrs, which were active during struggling, lay in the hindbrain and rostral spinal cord. We have used whole-cell patch recordings with neurobiotin to define their activity, properties, and anatomy (Fig. 4A). In particular, we needed to establish criteria to distinguish $\mathrm{dIN} r$ from other excitatory interneurons: the dINs whose characteristics we have defined previously (Li et al., 2006). Anatomically, they cannot be separated because both have fairly ventral somata with dendrites from the soma and an 
A

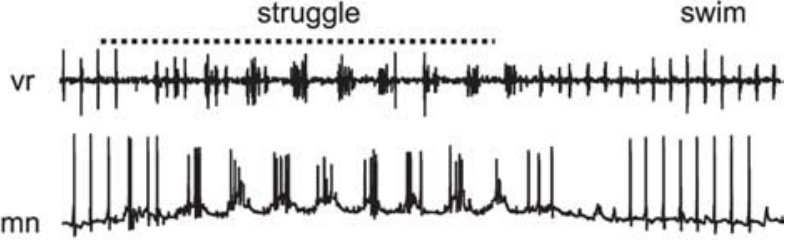

B

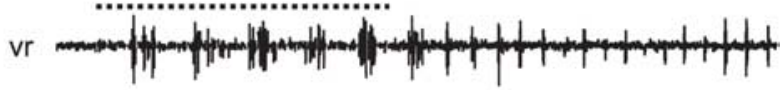

$\mathrm{CIN}$

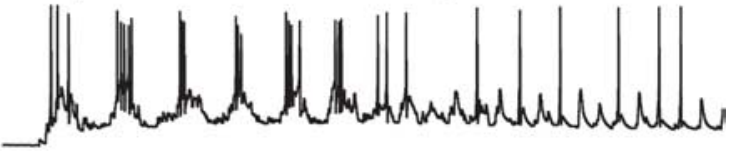

C

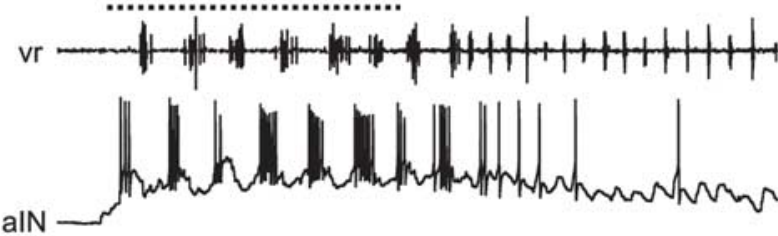

D
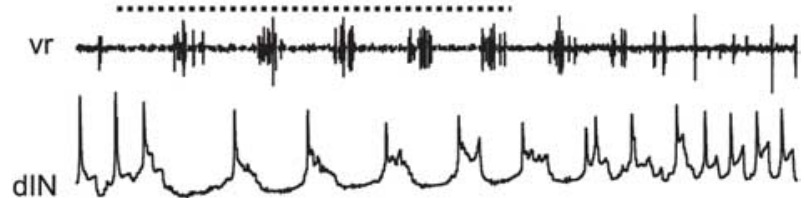

E
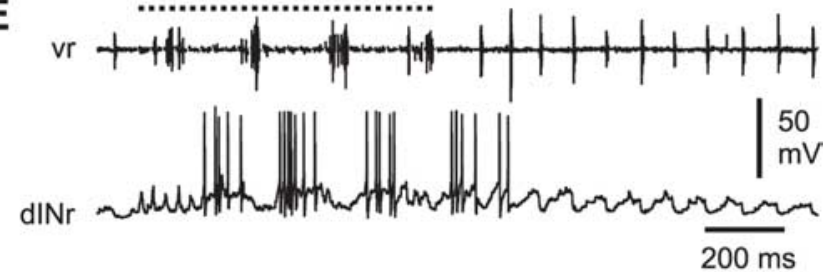

$\mathbf{F}$

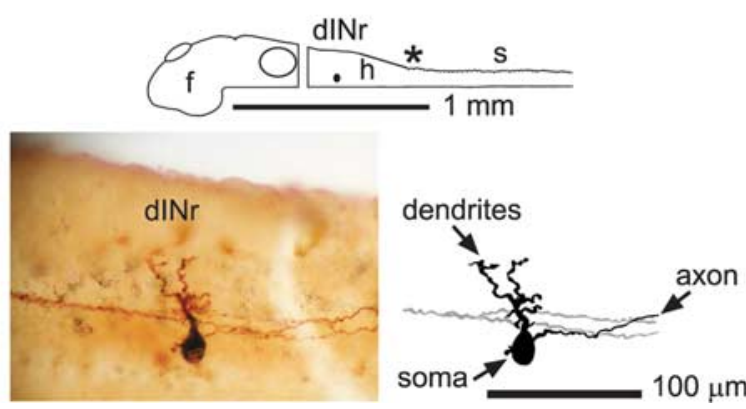

Figure 3. Neurons active during struggling. $\boldsymbol{A}, \mathrm{A}$ motoneuron that fires strongly during struggling, evoked by $40 \mathrm{~Hz}$ stimulation of the trunk skin (dotted line), and also fires reliably during swimming. $\boldsymbol{B}, \boldsymbol{C}, \mathrm{A} \subset \mathrm{CN}(\boldsymbol{B})$ and an aIN $(\boldsymbol{C})$ that are recruited to fire strongly during struggling; both fire unreliably during swimming. D, A dIN that fires weakly, one spike per cycle, during struggling, but fires reliably during swimming. $\boldsymbol{E}, \mathrm{A}$ dIN $r$ that is recruited to fire strongly during struggling but does not fire during swimming. $F$, Diagram, photomontage, and tracing to show the right-side dIN $r$ in $\boldsymbol{E}$ with prominent dorsal dendrites and descending axon (axons from another neuron are gray in diagram). Asterisk indicates obex.

ipsilateral descending axonal projection. Fortunately, there were clear physiological differences. In 15 dIN $r$ s firing $>3$ spikes per cycle in struggling, action potentials were significantly shorter $(0.81 \pm 0.17 \mathrm{~ms} ; n=15)$ than in dINs $(1.93 \pm 0.43 \mathrm{~ms} ; n=56$; $p<0.001$ ) (Fig. $4 B$ ). The trough of the AHP was also significantly sooner after the spike peak, compared with dINs ( $\mathrm{dIN} r, 1.7 \pm 0.5$ ms; dIN, $7.2 \pm 2.0 \mathrm{~ms} ; p<0.001)$. The dIN $r$ s fired highfrequency, nonadapting, repetitive spikes to depolarizing current injection (average maximum frequency observed, $129 \pm 55 \mathrm{~Hz}$ ) (Fig. 4C), whereas dINs only fire a single action potential. These features of narrow spike and repetitive firing were used to distinguish a further $16 \mathrm{dIN} r$ from other dINs, providing a total of 31 dINrs, which were then used to define the characteristics of this new group of interneurons.

Of 23 dIN $r$ s in 22 animals where fictive swimming was evoked by skin stimulation, 15 were completely silent (Fig. $4 D$ ), four fired action potentials sporadically on some swimming cycles, and only four fired reliably on most cycles. In 19 of the same animals where reliable struggling activity was seen in the ventral root, 15 of these dINrs were strongly active (Fig. $4 E$ ), firing three or more spikes per cycle (mean $7.1 \pm 3.0$ spikes per burst) at instantaneous frequencies up to $244 \mathrm{~Hz}$ (mean $180 \pm 45 \mathrm{~Hz} ; n=$ 12) and the remaining four were weakly active.

The mean dIN $r$ resting membrane potential was $-59.4 \pm 4.0$ $\mathrm{mV}(n=27)$, significantly more negative than $\operatorname{dINs}(-50.9 \pm 3.6$ $\mathrm{mV} ; n=56 ; p<0.001)$. Voltage responses to current injection around the resting membrane potential were linear and input resistances ranged from 147 to $1600 \mathrm{M} \Omega$ (median, $296 \mathrm{M} \Omega ; n=$ 22).

Paired recordings showed that dIN $r$ s excite more caudal ipsilateral neurons (Fig. 5A) $(n=16,10 \mathrm{mns}, 2 \mathrm{cINs}, 1$ aIN, $1 \mathrm{dIN} r$ and 2 unidentified CPG neurons). The EPSP latencies (range, $0.9-2.5 \mathrm{~ms}$; mean, $1.5 \pm 0.4 \mathrm{~ms} ; n=15$ ) were within the normal range seen for monosynaptic connections in this preparation (see above). The EPSP amplitudes lay in the range 1.1-8.8 $\mathrm{mV}$ (3.9 \pm $2.4 \mathrm{mV}, n=14)$. Bath application of the glutamate antagonists NBQX $(2.5-5 \mu \mathrm{M})$ and D-AP5 $(25-50 \mu \mathrm{M})$ in five recordings blocked the EPSPs suggesting that they were mediated by glutamate alone (Fig. 5B).

Electrical stimulation of the skin on either side of the body produced EPSPs in dINrs (Fig. 5D) at latencies short enough to indicate, at most, a disynaptic connection from sensory RohonBeard neurons (ipsilateral, $5.8 \pm 0.9 \mathrm{~ms}, n=7$; contralateral, $6.4 \pm 1.4, n=13$ ). Single skin stimuli very rarely evoked a dIN $r$ impulse. EPSPs showed evidence of summation at stimulus repetition rates and amplitudes sufficient to produce struggling but the first impulse was not usually evoked for several tens of ms (Figs. 3E, 4E, 5E).

$\mathrm{dIN} r$ somata lay in the hindbrain and rostral spinal cord 0.33 $1.21 \mathrm{~mm}$ caudal to the midbrain (Fig. 5C). Their multipolar somata lay in a ventral position with dendrites emerging laterally and dorsally from the soma (Figs. 3F, 4A). Each neuron had a descending axon extending from 0.23 to $1.83 \mathrm{~mm}$ caudally (Fig. $5 C$ ) (mean length, $0.98 \pm 0.09 \mathrm{~mm} ; n=26$ ) and lying in the ventral half of the cord marginal zone. In contrast to dINs, no dINrs had an ascending axon branch.

\section{Exploring a neuronal network to generate struggling}

We have now defined two new classes of neuron that are recruited during struggling (whose physiological properties are summarized in supplemental Table 1, available at www.jneurosci.org as supplemental material). The first class are sensory pathway ecINs, which are excited during repetitive skin stimulation and in turn excite neurons on the opposite side via their commissural axons. This explains how contralateral neurons can be active during struggling when only one side is stimulated. The second class form a new, physiologically distinct subset of excitatory interneurons mainly in the hindbrain with descending projections (dINrs) that are anatomically indistinguishable from the excita- 
tory interneurons active during swimming (dINs). DINrs are generally silent during swimming but are recruited during repetitive skin stimulation that leads to struggling. They excite ipsilateral neurons, apparently taking over the role of dINs, which are inactive or only weakly active during struggling. In contrast to dINs, dIN rs fire repetitively to current and during struggling. They produce simple glutamate mediated excitation, whereas dINs corelease glutamate and acetylcholine ( $\mathrm{Li}$ et al., 2004b). We now need to ask whether these neurons can connect to form a network capable of generating the slow bursting pattern of struggling during repeated sensory stimulation to the skin.

The approach we have taken to explore how the reconfigured caudal hindbrain and spinal cord network generates a slower pattern of rhythmic motor bursts suitable for struggling is to build a simple halfcenter network model where small numbers of model neurons represent longitudinal columns of neurons. This model does not have a length dimension as we have not investigated the characteristic tail-to-head progression of activity during struggling (Kahn and Roberts, 1982). The model incorporates results from the present study and other previous evidence from whole-cell patch recordings defining the specific properties of different neuron classes and their synaptic connections [Sautois et al. (2007), their Table 1] (supplemental Table 2, available at www. jneurosci.org as supplemental material). One feature of the neuron properties is that most, including the two new classes of ecINs and dINrs, typically fire repetitively if depolarized by current injection. This was not the case with our earlier models of swimming (Tunstall et al., 2002). In life, the excitation to drive continued struggling comes from the repetitive activity of skin sensory RB neurons, usually on both sides of the body (Soffe, 1997). Ipsilateral to the stimulus, the evidence indicates that excitation to CPG neurons comes directly from RBs (Li et al., 2004a). Contralateral excitation comes indirectly via the ecINs described above. Because there is still uncertainty about the details of the sensory pathways activating struggling, we have simplified them to two driver neurons, one activated $20 \mathrm{~ms}$ after the other, and producing steady, NMDAR-mediated excitation to each side. Our aim was to find whether a simple spinal network could convert this steady drive into a pattern capturing the essence of struggling: slow $(2-10 \mathrm{~Hz})$, alternating bursts.

A spinal network model was made which included 3 of each type of CPG neuron active during struggling (aINs, cINs, dINrs, and mns) (Fig. 6A). The three neurons of each type were in some cases given slightly different input and output synaptic strengths so that their firing was not synchronous and therefore more like a larger population. Occasionally, activation of the two driver neurons led to alternating left-right bursting activity within the range seen during struggling (Fig. 6B). To search for stable areas of bursting we varied the synaptic strengths of the excitation from the driver neurons and the reciprocal inhibition from cINs. Alternating bursting occurred only at certain critical combinations of the synaptic strengths. In most cases, one side became active and suppressed activity on the other side indefinitely (for more examples of model output, see supplemental material, available at www.jneurosci.org). This suggested that the network lacked a satisfactory burst termination mechanism. We expected that the recurrent inhibition provided by aINs, which fire strongly during struggling, would terminate bursts, but it did not appear sufficient for this, even when varied over a range of synaptic strengths (data not shown). Spike firing adaptation could also provide a burst termination mechanism, but there is no evidence for this property in the firing responses of excitatory dINrs (Fig. 4C) or of reciprocally inhibitory cINs (Aiken et al., 2003; Li et al., 2004c). We therefore investigated the possibility that burst termination during struggling might depend on synaptic depression in reciprocal inhibition.

\section{Evidence for synaptic depression in reciprocal inhibition}

To investigate whether depression of reciprocal inhibition could act as a burst termination mechanism in the struggling network, we examined the plasticity of synapses from reciprocal inhibitory cINs. Our aim was to stimulate cINs, which are the only known inhibitory neurons with commissural axonal projections (Dale, 1985), and record the IPSCs that they produced in neurons on the opposite side of the spinal cord. Glutamate and ACh excitation 


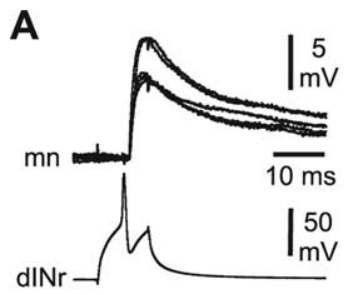

B

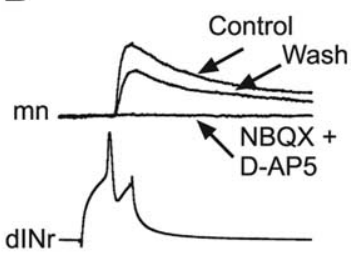

C

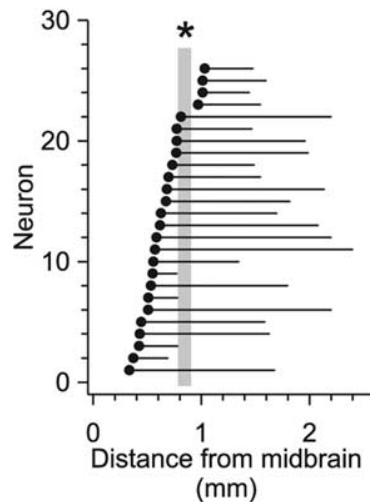

D

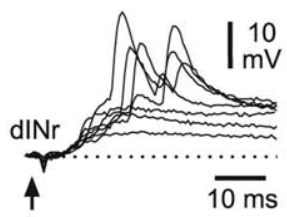

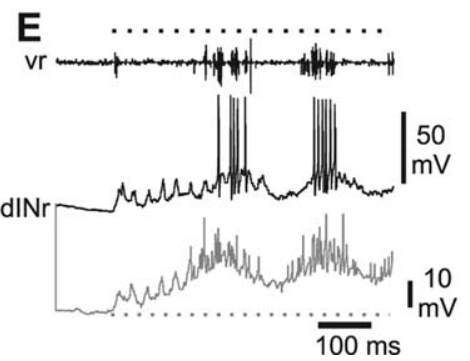

was blocked with NBQX (5 $\mu \mathrm{M})$, D-AP5 $(50 \mu \mathrm{M})$, and dihydro- $\beta$-erythroidine (2 $\mu \mathrm{M})$. We then recorded from CPG neurons under voltage clamp, at a holding potential of $0 \mathrm{mV}$, while the opposite side of the cord was stimulated with a glass suction electrode. In four of seven neurons recorded, stimulation evoked compound IPSCs in the contralateral CPG neuron. These IPSCs were reliable and showed no significant depression at a typical swimming frequency of $20 \mathrm{~Hz}$; however, they became significantly depressed at $100 \mathrm{~Hz}$, typical of the frequencies at which cINs fire during struggling bursts (see above) (Fig. $7 A-C$ ). These results were corroborated by making paired recordings from single cINs on one side of the cord with CPG neurons on the other ( $n=5$ pairs). In 3 cases cIN action potentials caused by intracellular current injection evoked unitary IPSCs in the contralateral CPG neuron. These unitary IPSCs were less reliable than the compound IPSCs but, once again, they showed significant depression at $100 \mathrm{~Hz}$ but not at $20 \mathrm{~Hz}$ (Fig. $7 D-F$ ).

\section{Modeling a neuronal network with synaptic depression}

The evidence from recordings of reciprocal inhibitory synaptic currents showed that they depress when cINs fire at the frequencies that occur during struggling. As originally proposed by Brown (1911) this depression of inhibition could act as a burst termination mechanism in a halfcenter model, because weakening inhibition could allow antagonistic reciprocal inhibitory neurons to start firing and terminate the current burst. We therefore introduced depression into the model cIN synapses and tested that this produced a suitable reduction in currents when stimulated at struggling frequencies (Fig. $7 G, H)$. When depression at reciprocal inhibitory cIN synapses was included in the model network, activation led to reliable alternating bursting at frequencies typical of normal struggling (Fig. 6C). This struggling-like activity was stable over a range of excitatory and inhibitory synaptic strengths. The range of effective depression levels is illustrated in Figure $6 D$. Broadly, as depression became strong or weak, the excitatory and inhibitory synaptic strengths producing stable bursting became very constrained. We found that the rhythmic burst activity depended critically on the ability of the excitatory dIN $r$ s to fire repetitively to current because it failed if they were replaced with dINs which only fire a single spike to sustained injected current.
Figure 6. A half-center model of the struggling network in the caudal hindbrain and rostral spinal cord. $\boldsymbol{A}$, Diagram of the model network. The large squares are the half-centers. Circles represent neurons where $\$ 1$ and S2 are the sensory driver neurons. Triangles are excitatory synapses and black dots inhibitory synapses made onto all neurons within one half-center. Motoneurons are coupled by electrical junctions. $\boldsymbol{B}$, Alternating bursting in one example of each neuron type during continuous NMDAR mediated excitation produced by the driver neurons (S1 and S2) in a network without synaptic depression. $\boldsymbol{C}$, Stable bursting activity when $\mathrm{IN}$ synapses show depression $(\alpha=0.5 ; \beta=38)$. $\boldsymbol{D}$, Graphs showing shaded areas of stable alternating struggling over a range of synaptic strengths in the driving NMDA mediated excitation (Exc) from the driver neurons S1 and S2 and reciprocal inhibition (Inh) from the cINs for four different values of the depression in reciprocal inhibition (depression increases from $\alpha=0.4$ to $\alpha=0.7)$.

\section{B}

C

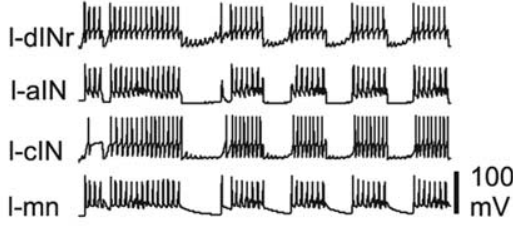

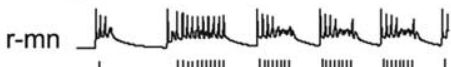

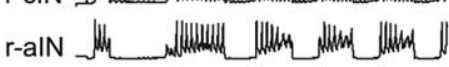

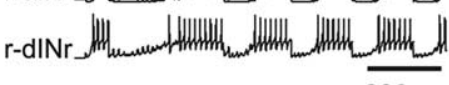

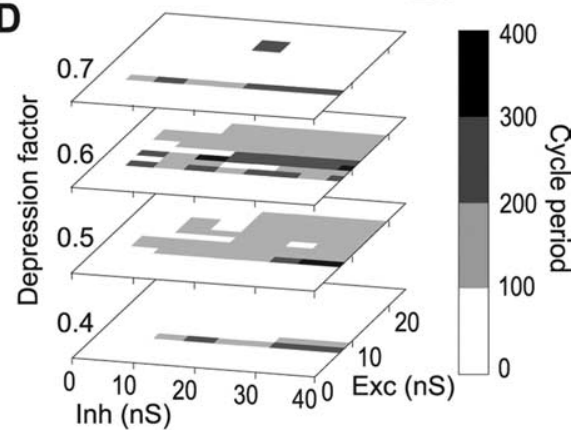


A

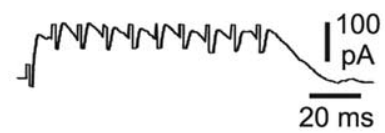

B

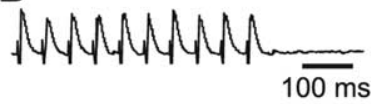

D
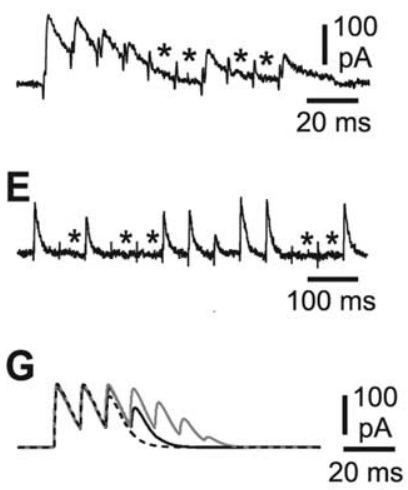

C

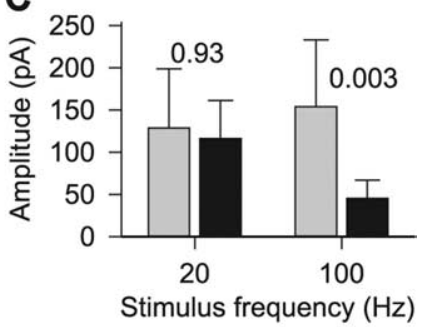

$\mathbf{F}$

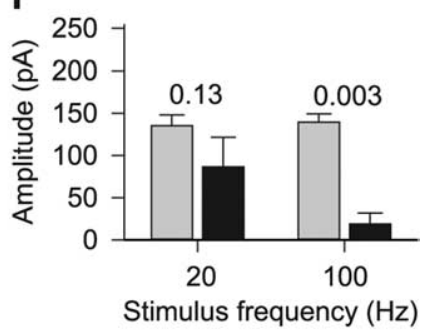

H

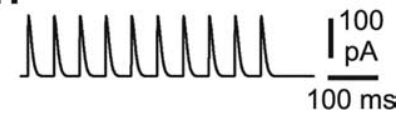

Figure 7. Depression of reciprocal inhibition at struggling frequencies. $\boldsymbol{A}, \boldsymbol{B}$, Compound IPSCs evoked in a dIN by stimulation of the spinal cord on the opposite side at $100 \mathrm{~Hz}(\boldsymbol{A})$ and 20 $\mathrm{Hz}(\boldsymbol{B})$. C, Measurement of the amplitude of the first (gray) and tenth (black) IPSC in a sequence show significant depression at $100 \mathrm{~Hz}$, but not at $20 \mathrm{~Hz}$ ( $p$ values are indicated; $n=4$ neurons, 5 trials per neuron). $\boldsymbol{D}, \boldsymbol{E}$, Unitary IPSC s evoked in a $\mathrm{mn}$ by intracellular stimulation of a single cIN on the opposite side of the spinal cord at $100 \mathrm{~Hz}(\boldsymbol{D})$ and $20 \mathrm{~Hz}(\boldsymbol{E})$. Asterisks indicate failures. $\boldsymbol{F}$, Measurement of the amplitude of the first (gray) and tenth (black) IPSC in a sequence again shows significant depression at $100 \mathrm{~Hz}$, but not at $20 \mathrm{~Hz}$ ( $p$ values are indicated; $n=3$ pairs, 10 trials per pair). G, IPSCs at a model $\mathrm{CIN}$ to dINr reciprocal inhibition synapse with depression [recovery time constant, $\beta=38 \mathrm{~ms}$; plasticity factor, $\alpha=0.4$ (gray), 0.5 (black), and 0.6 (dashed line)]. Depression in currents is clear at $100 \mathrm{~Hz}$ but not at $20 \mathrm{~Hz}(\boldsymbol{H})$.

\section{Discussion}

The process of reconfiguration of the hatchling Xenopus tadpole spinal circuitry that occurs when skin stimulation selects struggling rather than swimming involves different kinds of change. The most obvious is a change in the neurons participating in rhythm generation: the CPGs for swimming and struggling have shared elements, but there is also selective recruitment (Fig. 8). It was originally concluded that a switch to struggling involved recruitment only within classes that already participate in swimming (recurrent inhibitory aINs, reciprocal inhibitory cINs, and motoneurons) (Soffe, 1993). However, we now show that this conclusion was incorrect and that the switch involves additional recruitment of two newly defined excitatory neuron classes: the ecINs and dINrs. Furthermore, the excitatory interneurons that drive swimming (dINs) show only weak activity while the dorsolateral ascending (dla) and dlc sensory interneurons that activate swimming are actively inhibited (Soffe, 1993; Li et al., 2002, 2004a). Why are some neurons common to both swimming and struggling CPGs, whereas others are circuit specific?

The first place in which we provide evidence for circuitspecific recruitment is in the sensory pathway from the trunk skin. The two sensory interneuron classes implicated in the initiation of swimming, dlc and dla sensory interneurons, fire in the early stages of repetitive stimulation and may play a role in initiating struggling, but are silent once struggling is established. In contrast, during repeated skin stimulation a new type of sensory

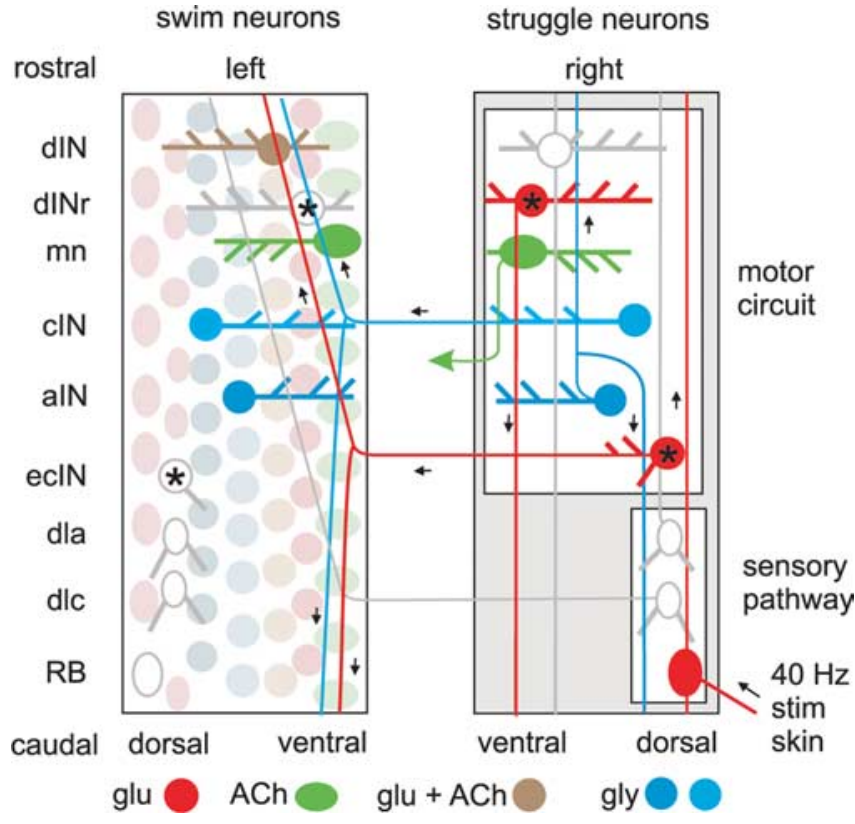

Figure 8. Summary diagram of the Xenopus tadpole hindbrain and spinal cord as if opened like a book along the dorsal midline to show the established neuron classes and the two new classes defined in this study (asterisks). The colored neurons on the right are active during struggling, those on the left during swimming. Neuron somata are arranged in loosely organized longitudinal columns (see left side). Each neuron has one or two axons (thinner lines) which make en passant synapses onto dendrites (thicker lines). Soma color indicates the transmitter used. Short arrows indicate signa flow.

pathway interneuron, ecIN, is recruited and can fire throughout struggling. At present, the significance of this first case of circuitspecific recruitment is unclear. Our limited evidence does not suggest that the postsynaptic targets of ecINs are different from those of dlc sensory interneurons. It seems likely that there needs to be an uncoupling of the firing patterns among the neurons active in struggling. The glutamatergic connections from skin sensory RB neurons to dlc interneurons are very strong and dominated by AMPAR ( $\mathrm{Li}$ et al., 2003) to produce reliable, short latency, one-to-one firing. The sensory input from RB to ecIN interneurons appears to be more variable and generally weaker. Summation of smaller EPSPs might allow excitation of the struggling CPG to be uncoupled from the exact temporal pattern of stimulation and sensory discharge.

A characteristic difference between the motor patterns for swimming and struggling is that in swimming neurons fire a single spike on each cycle alternating between the two sides, whereas struggling involves alternating bursts of spikes. We should therefore expect that neurons suitable to drive and coordinate struggling would fire bursts of discharge. We now know that most swimming CPG neurons fire repetitively when depolarized above threshold (Sautois et al., 2007). This does not cause a problem during swimming as their firing is driven by fast EPSPs on each cycle that depolarize the neurons sufficiently briefly that they will only fire a single spike per cycle (Li et al., 2006). During struggling, the extended period of excitation will then produce repetitive firing in the same neurons. Their different firing patterns during swimming and struggling will therefore simply reflect the different durations of underlying excitatory synaptic drive. There seems to be no requirement for these neurons to be circuit specific.

In contrast, the excitatory neurons (dINs) that drive swimming and produce the brief excitation in swimming CPG neurons 
on each cycle apparently need different firing properties. These dINs fire only a single long spike when depolarized. Modeling studies have shown than if they are replaced by repetitive firing neurons, swimming becomes much less robust (Sautois et al., 2007). During struggling, the dINs are apparently replaced by dINrs: a new class of excitatory interneurons that, while morphologically like the dINs, are physiologically distinct having short duration spikes and the property of firing repetitively when depolarized. These neurons are typically silent during swimming, but are recruited during struggling. Neuromodulation of cellular and synaptic properties is widespread in invertebrates as a means of selecting particular patterns from multifunctional networks (Marder et al., 2005). From this, one might expect dIN properties to be modulated to allow them to fire repetitive bursts during a switch to struggling. Instead, they appear to be largely replaced in the struggling CPG by burst-firing dINrs. Perhaps the speed of switching between swimming and struggling in response to continuous sensory stimulation means that modulation is not an option. We conclude that there is selective recruitment of different types of excitatory CPG neuron with different firing properties between swimming and struggling: dIN for swimming and $\mathrm{dIN} r$ for struggling.

Patterns of recruitment among excitatory interneurons have been described previously in zebrafish larvae (Bhatt et al., 2007; McLean et al., 2007). These animals, which have several different locomotor responses (Budick and O’Malley, 2000), show selective recruitment of excitatory interneurons during fast escape and slower swimming movements (Ritter et al., 2001). Like neurons recruited selectively for swimming and scratching in turtles (Berkowitz, 2007), these zebrafish interneurons are morphologically distinct. However, selective recruitment for different zebrafish larva responses has now also been described within a single morphological group of alx excitatory interneurons (Kimura et al., 2006). It remains to be determined whether the anatomically homogeneous but physiologically distinct tadpole dINs and dINrs, active during faster swimming and slower struggling respectively, are related to these spinal alx neurons, which also have ipsilateral descending axons.

An essential feature of a motor pattern based on rhythmic alternating bursts of discharge is that each burst must terminate. This requirement was highlighted explicitly by Brown (1911), who proposed a "depreciation of inhibition" as an underlying mechanism (Brown, 1914). Our evidence shows exactly this process in the form of depression of the reciprocal inhibitory synapses from cINs, occurring at cIN firing frequencies like those seen during struggling bursts. The modeling shows that this represents a sufficient mechanism to allow robust generation of rhythmic alternating bursts. Importantly, the cIN synaptic depression is not seen at lower firing rates, like those seen during swimming. In this sense therefore, it represents a contextdependent plasticity: making no contribution to swimming pattern generation, but becoming a significant mechanism after network reconfiguration for struggling simply as a result of altered cIN firing frequency.

Investigations in the lamprey have highlighted other possible mechanisms for burst termination. Build-up of recurrent inhibition is one plausible mechanism (Buchanan and Grillner, 1987; Buchanan, 1999) but is not the only factor (Grillner et al., 1991; Hellgren et al., 1992). $\mathrm{Ca}^{2+}$ dependent $\mathrm{K}^{+}\left(\mathrm{K}_{\mathrm{Ca}}\right)$ conductances appear to play a more significant role by producing spike firing adaptation in inhibitory interneurons (el Manira et al., 1994). $\mathrm{K}_{\mathrm{Ca}}$ conductances are also thought to be key to termination of mammalian respiratory bursts (Kosmidis et al., 2004; Feldman and Del Negro, 2006). In the tadpole, our present modeling sug- gests that recurrent inhibition from aINs is unable to produce reliable struggling and we see no evidence for significant spike firing adaptation in CPG neurons. In older Xenopus larvae, some neurons fire bursts of discharge during swimming (Sillar et al., 1991, 1992). Here, a fast activating $\mathrm{K}_{\mathrm{Ca}}$ conductance plays a role in swimming burst termination, but this conductance is apparently mostly absent from the younger tadpoles used here (Sun and Dale, 1998) (but see Wall and Dale, 1995). Although other contributory mechanisms could exist therefore, and in the absence of critical experimental tests, our evidence suggests that synaptic depression of reciprocal inhibition may provide a sufficient burst termination mechanism that allows the struggling rhythm to be generated.

If, as our modeling suggests, recurrent inhibitory aINs are not primarily concerned with burst termination, their strong recruitment during struggling ( $\mathrm{Li}$ et al., 2002) may emphasize a need during reconfiguration to gate out the dlc and dla sensory interneurons, which initiate swimming (Soffe, 1993; Li et al., 2002, 2004a). During struggling, recurrent inhibitory aINs and reciprocal inhibitory cINs are broadly coactive, so struggling neurons receive inhibition throughout each struggling cycle ( $\mathrm{Li}$ et al., 2002). Because the excitatory dINrs and commissural ecINs are also active together, CPG neurons will also receive excitation throughout each cycle of struggling (Soffe, 1993). Although we are not suggesting that rhythmic firing in struggling is driven by a balanced increase in excitation and inhibition, as recently described for turtle scratching (Berg et al., 2007), the synaptic drive to struggling neurons is clearly not a simple alternation of excitation and inhibition. The combination of excitation and inhibition underlying bursts of firing may provide a mechanism for controlling their strength.

In conclusion, we have been able to take advantage of the simplicity of the hatchling tadpole hindbrain and spinal cord to define the pattern of recruitment of neurons, both within and between neuron classes, that occurs during network reconfiguration between circuits for swimming and struggling. It remains possible that other physiological classes of neurons may emerge, but this will not change the basic conclusion that reconfiguration here involves a combination of core, shared neurons and additional selective recruitment. At the same time, we have been able to show context-dependent synaptic plasticity: a depression of reciprocal inhibitory synaptic transmission that is not seen during swimming, but plays a key role during struggling. Such depression of reciprocal inhibition was proposed by Brown (1914) and has been widely explored in theoretical studies (Taylor et al., 2002), but we are not aware of any other similarly direct experimental support for this long-standing proposal.

\section{References}

Aiken SP, Kuenzi FM, Dale N (2003) Xenopus embryonic spinal neurons recorded in situ with patch-clamp electrodes-conditional oscillators after all? Eur J Neurosci 18:333-343.

Bekoff A, Nusbaum MP, Sabichi AL, Clifford M (1987) Neural control of limb coordination. I. Comparison of hatching and walking motor output patterns in normal and deafferented chicks. J Neurosci 7:2320-2330.

Berg RW, Alaburda A, Hounsgaard J (2007) Balanced inhibition and excitation drive spike activity in spinal half-centers. Science 315:390-393.

Berkinblit MB, Deliagina TG, Feldman AG, Gelfand IM, Orlovsky GN (1978) Generation of scratching. II. Nonregular regimes of generation. J Neurophysiol 41:1058-1069.

Berkowitz A (2002) Both shared and specialized spinal circuitry for scratching and swimming in turtles. J Comp Physiol 188:225-234.

Berkowitz A (2005) Physiology and morphology indicate that individual spinal interneurons contribute to diverse limb movements. J Neurophysiol 94:4455-4470. 
Berkowitz A (2007) Spinal interneurons that are selectively activated during fictive flexion reflex. J Neurosci 27:4634-4641.

Bhatt DH, McLean DL, Hale ME, Fetcho JR (2007) Grading movement strength by changes in firing intensity versus recruitment of spinal interneurons. Neuron 53:91-102.

Briggman KL, Kristan Jr WB (2006) Imaging dedicated and multifunctional neural circuits generating distinct behaviors. J Neurosci 26:10925-10933.

Brown TG (1911) The intrinsic factor in the act of progression of the mammal. Proc R Soc Lond B Biol Sci 84:308-319.

Brown TG (1914) On the nature of the fundamental activity of the nervous centres; together with an analysis of the conditioning of rhythmic activity in progression, and a theory of the evolution of function in the nervous system. J Physiol (Lond) 48:18-46.

Buchanan JT (1999) The roles of spinal interneurons and motoneurons in the lamprey locomotor network. Prog Brain Res 123:311-321.

Buchanan JT, Grillner S (1987) Newly identified "glutamate interneurons" and their role in locomotion in the lamprey spinal cord. Science 236:312-314

Budick SA, O'Malley DM (2000) Locomotor repertoire of the larval zebrafish: swimming, turning and prey capture. J Exp Biol 203:2565-2579.

Carter MC, Smith JL (1986) Simultaneous control of two rhythmical behaviors. I. Locomotion with paw-shake response in normal cat. J Neurophysiol 56:171-183.

Clarke JDW, Hayes BP, Hunt SP, Roberts A (1984) Sensory physiology, anatomy and immunohistochemistry of Rohon-Beard neurones in embryos of Xenopus laevis. J Physiol (Lond) 348:511-525.

Currie SN, Stein PS (1989) Interruptions of fictive scratch motor rhythms by activation of cutaneous flexion reflex afferents in the turtle. J Neurosci 9:488-496.

Dale N (1985) Reciprocal inhibitory interneurones in the Xenopus embryo spinal cord. J Physiol (Lond) 363:61-70.

el Manira A, Tegner J, Grillner S (1994) Calcium-dependent potassium channels play a critical role for burst termination in the locomotor network in lamprey. J Neurophysiol 72:1852-1861.

Feldman JL, Del Negro CA (2006) Looking for inspiration: new perspectives on respiratory rhythm. Nature Revs Neurosci 7:232-242.

Getting PA (1989) Emerging principles governing the operation of neural networks. Annu Rev Neurosci 12:185-204.

Getting PA, Dekin MS (1985) Mechanisms of pattern generation underlying swimming in Tritonia. IV. Gating of central pattern generator. J Neurophysiol 53:466-480.

Grillner S, Wallen P, Brodin L, Lansner A (1991) Neuronal network generating locomotor behavior in lamprey: circuitry, transmitters, membrane properties, and simulation. Ann Rev Neurosci 14:169-199.

Hellgren J, Grillner S, Lansner (1992) A computer simulation of the segmental neural network generating locomotion in lamprey by using populations of network interneurons. Biol Cybern 68:1-13.

Jing J, Weiss KR (2002) Interneuronal basis of the generation of related but distinct motor programs in Aplysia: implications for current neuronal models of vertebrate intralimb coordination. J Neurosci 22:6228-6238.

Kahn JA, Roberts A (1982) The neuromuscular basis of rhythmic struggling movements in embryos of Xenopus laevis. J Exp Biol 99:197-205.

Kahn JA, Roberts A, Kashin SM (1982) The neuromuscular basis of swimming movements in embryos of the amphibian Xenopus laevis. J Exp Biol 99:175-184.

Kimura Y, Okamura Y, Higashijima S (2006) alx, a zebrafish homolog of Chx10, marks ipsilateral descending excitatory interneurons that participate in the regulation of spinal locomotor circuits. J Neurosci 26:5684-5697.

Kosmidis EK, Pierrefiche O, Vibert JF (2004) Respiratory-like rhythmic activity can be produced by an excitatory network of non-pacemaker neuron models. J Neurophysiol 92:686-699.

Li WC, Perrins R, Soffe SR, Yoshida M, Walford A, Roberts A (2001) Defining classes of spinal interneuron and their axonal projections in hatchling Xenopus laevis tadpoles. J Comp Neurol 441:248-265.

Li WC, Soffe SR, Roberts A (2002) Spinal inhibitory neurons that modulate cutaneous sensory pathways during locomotion in a simple vertebrate. J Neurosci 22:10924-10934.

Li WC, Soffe SR, Roberts A (2003) The spinal interneurons and properties of glutamatergic synapses in a primitive vertebrate cutaneous flexion reflex. J Neurosci 23:9068-9077.
Li WC, Soffe SR, Roberts A (2004a) Dorsal spinal interneurons forming a primitive, cutaneous sensory pathway. J Neurophysiol 92:895-904.

Li WC, Soffe SR, Roberts A (2004b) Glutamate and acetylcholine corelease at developing synapses. Proc Natl Acad Sci USA 101:15488-15493.

Li WC, Soffe SR, Roberts A (2004c) A direct comparison of whole cell patch and sharp electrodes by simultaneous recording from single spinal neurons in frog tadpoles. J Neurophysiol 92:380-386.

Li WC, Higashijima S, Parry DM, Roberts A, Soffe SR (2004d) Primitive roles for inhibitory interneurons in developing frog spinal cord. J Neurosci 24:5840-5848.

Li WC, Soffe SR, Wolf E, Roberts A (2006) Persistent responses to brief stimuli: feedback excitation among brainstem neurons. J Neurosci 26:4026-4035.

Lieske SP, Thoby-Brisson M, Telgkamp P, Ramirez JM (2000) Reconfiguration of the neural network controlling multiple breathing patterns: eupnea, sighs and gasps [see comment]. Nat Neurosci 3:600-607.

Marder E, Bucher D, Schulz DJ, Taylor AL (2005) Invertebrate central pattern generation moves along. Curr Biol 15:R685-R698.

McLean DL, Fan J, Higashijima S, Hale ME, Fetcho JR (2007) A topographic map of recruitment in spinal cord. Nature 446:71-75.

Meyrand P, Simmers J, Moulins M (1991) Construction of a patterngenerating circuit with neurons of different networks. Nature 351:60-63.

Mortin LI, Stein PS (1989) Spinal cord segments containing key elements of the central pattern generators for three forms of scratch reflex in the turtle. J Neurosci 9:2285-2296.

Popescu IR, Frost WN (2002) Highly dissimilar behaviors mediated by a multifunctional network in the marine mollusk Tritonia diomedea. J Neurosci 22:1985-1993.

Ritter DA, Bhatt DH, Fetcho JR (2001) In vivo imaging of zebrafish reveals differences in the spinal networks for escape and swimming movements. J Neurosci 21:8956-8965.

Roberts A, Walford A, Soffe SR, Yoshida M (1999) Motoneurons of the axial swimming muscles in hatchling Xenopus tadpoles: features, distribution, and central synapses. J Comp Neurol 411:472-486.

Sautois B, Soffe SR, Li W-C, Roberts A (2007) Role of type-specific neuron properties in a spinal cord motor network. J Comput Neurosci 23:59-77.

Sillar KT, Wedderburn JF, Simmers AJ (1991) The development of swimming rhythmicity in post-embryonic Xenopus laevis. Proc R Soc Lond B Biol Sci 246:147-153.

Sillar KT, Wedderburn JF, Simmers AJ (1992) Modulation of swimming rhythmicity by 5 -hydroxytryptamine during post-embryonic development in Xenopus laevis. Proc R Soc Lond B Biol Sci 250:107-114.

Soffe SR (1990) Active and passive membrane properties of spinal cord neurons that are rhythmically active during swimming in Xenopus embryos. Eur J Neurosci 2:1-10.

Soffe SR (1991) Triggering and gating of motor responses by sensory stimulation: behavioural selection in Xenopus embryos. Proc R Soc Lond B Biol Sci 246:197-203.

Soffe SR (1993) Two distinct rhythmic motor patterns are driven by common premotor and motor neurons in a simple vertebrate spinal cord. J Neurosci 13:4456-4469.

Soffe SR (1996) Motor patterns for two distinct rhythmic behaviors evoked by excitatory amino acid agonists in the Xenopus embryo spinal cord. J Neurophysiol 75:1815-1825.

Soffe SR (1997) The pattern of sensory discharge can determine the motor response in young Xenopus tadpoles. J Comp Physiol A Neuroethol Sens Neural Behav Physiol 180:711-715.

Stein PSG (2005) Neuronal control of turtle hindlimb motor rhythms. J Comp Physiol A Neuroethol Sens Neural Behav Physiol 191:213-229.

Sun QQ, Dale N (1998) Differential inhibition of N and P/Q Ca ${ }^{2+}$ currents by 5-HT1A and 5-HT1D receptors in spinal neurons of Xenopus larvae. J Physiol (Lond) 510:103-120.

Taylor AL, Cottrell GW, Kristan WB (2002) Analysis of oscillations in a reciprocally inhibitory network with synaptic depression. Neural Comput 14:561-581.

Tunstall MJ, Roberts A, Soffe SR (2002) Modelling inter-segmental coordination of neuronal oscillators: synaptic mechanisms for uni-directional coupling during swimming in Xenopus tadpoles. J Comput Neurosci 13:143-158.

Wall MJ, Dale N (1995) A slowly activating $\mathrm{Ca}^{2+}$-dependent $\mathrm{K}^{+}$current that plays a role in termination of swimming in Xenopus embryos. J Physiol (Lond) 487:557-572. 